\title{
String Structures and the Index of the Dirac-Ramond Operator on Orbifolds*
}

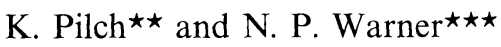 \\ Department of Mathematics, Massachusetts Institute of Technology, Cambridge, MA 02139, USA
}

\begin{abstract}
We discuss the relationship between "string structures" and the topological class $\lambda \in H^{4}(M, \mathbf{Z})$ on non-simply connected manifolds. We also investigate to what extent the index formula for the Dirac-Ramond operator detects the class, $\lambda$.
\end{abstract}

\section{Introduction}

It is now evident that the Dirac-Ramond operator [1] plays an important role in physics and mathematics. In physics it was shown that the anomaly generating function in string theory $[2,3]$ could be derived as the character valued index of this operator $[4,5]$. In mathematics a similar construction provided an explicit realization of the elliptic cohomology and was introduced to prove certain vanishing theorems [6-9] conjectured by Witten [10]. In fact by studying automorphisms of the Dirac-Ramond operator these can be now proven more directly $[5,11,12]$.

This interplay between physics and mathematics resulted in a rather unusual chronological order for the discovery of the properties of the Dirac-Ramond operator. For example, the index formula was known even before the operator itself had been rigorously defined ${ }^{1}$, and in particular, before the generalization of spin-structure had been properly understood.

In order to define a Dirac operator on a manifold, $M$, and hence discuss its index, the manifold must have a spin-structure. This is a topological restriction which is equivalent to requiring that the second Stiefel-Whitney class $w_{2}(M) \in H^{2}\left(M, \mathbf{Z}_{2}\right)$ vanishes. Physically, one can think of this like a Dirac quantization condition in the presence of the Dirac monopoles. On certain

\footnotetext{
* Supported by the NSF Grant \#84-07109 and by the U.S. Department of Energy (D.O.E.) under contract \#DE-AC02-76ER03069

$\star \star$ On leave from the University of Wroclaw, Wroclaw, Poland

$\star \star \star$ Present address: Theory Division, CERN, Geneva

1 In fact, there is still no precise definition of the general Dirac-Ramond operator. The operator used in [12] is most probably equivalent to the high temperature limit of the operator on the loop space
} 
manifolds (with $w_{2} \neq 0$ ) fermions naturally have wrong "charge" with respect to the affine connection. In some cases this can be corrected by coupling to a topologically nontrivial gauge field. This introduces the generalized spin structure, also called the $\operatorname{spin}^{C}$-structure. On a manifold $M$ with a spin structure, or a generalized spin structure one can define the Dirac operator coupled to gauge and gravitational fields.

The index of the operator is then given by the Atiyah-Singer theorem [13] as

$$
\text { Ind } D=\int_{M}[\hat{A}(R) \operatorname{ch}(F)]_{\text {top form }}
$$

where $\hat{A}(R)$ is the Dirac genus, and $\operatorname{ch}(F)$ is the Chern character of $F$ in the corresponding representation.

It is important to notice that the index formula (by which we will mean the integrand in the right-hand-side of (1.1)) is a set of curvature densities to be integrated over $M$, and therefore it makes perfect sense even if the manifold has no spin structure, or generalized spin structure, or if the appropriate Dirac quantization condition is not satisfied. However, if the integral fails to be an integer then the spinor fields in a given representation of the gauge group are obviously ill-defined. For example the $\hat{A}$-genus integrated over the even dimensional complex projective space $C P^{2 n}$ gives $(-1)^{n}\left((2 n) ! / 2^{4 n}(n !)^{2}\right)$ which is never an integer. The converse need not be true, i.e., if the index formula yields an integer, a spin structure, or a generalized spin structure does not necessarily exist.

In this paper we will study the corresponding issues in string theory. First we carefully examine the notion of "string structure" as described in [14] and find that when $M$ is not simply connected one needs to tighten up the definition by requiring that the string structure also respects the $\operatorname{Diff}^{+}\left(S^{1}\right)$ (the superscript ' + ' indicates oriented diffeomorphisms) action on the loop of the string. Once this is done we believe that the conclusions of [14] are correct in that the topological obstruction to the existence of the string structure is the $\lambda$-class ${ }^{2}$. The second part of this paper investigates to what extent the character valued index formula for the Dirac-Ramond operator detects the $\lambda$-class.

The class, $\lambda$, belongs in $H^{4}(M, \mathbf{Z})$ (note that this is the integer cohomology of $M$ ), and is related to the chiral and global anomalies on the string world-sheet. In general $\lambda$ has two parts, the free part, $\lambda^{\text {free }}$, which can be expressed in terms of curvatures on the manifold, and the torsion part $^{3}, \lambda^{\text {tor }}$, which cannot be written in terms of local quantities. However, on a large class of physically interesting spaces, for example on orbifolds, $\lambda^{\text {tor }}$ can be calculated explicitly. Also the existence of string structure in these spaces can be established directly without using computations in cohomology. It is by considering such a space that we are able to establish precisely how to properly modify the definition of string structure in [14]. Having done this, we generalize the index formula to orbifolds, which we

\footnotetext{
2 It is plausible that there may be implicit assumptions about $M$ in [14], like being simply connected, that allow to relate the obstruction in $H^{2}(L M, U(1))$ to $\lambda \in H^{4}(M, \mathbf{Z})$ by using the evaluation map $e: S^{1} \times L M \rightarrow M$ and integrating over $S^{1}$.

3 To be precise, the torsion part is unambiguously defined only when the free part vanishes
} 
will take to be an arbitrary manifold (not necessarily flat) divided by a discrete symmetry group, $\Gamma$. The $\lambda$-class for such a space can be schematically written as

$$
\lambda=\lambda_{4}[M]+\lambda_{2}[M] \lambda_{2}[\Gamma]+\lambda_{4}[\Gamma],
$$

where $\lambda_{4}[M]$ is the obstruction on the manifold itself, $\lambda_{4}[\Gamma]$ is related to twists at the critical points of the group action, and $\lambda_{2}[M] \lambda_{2}[\Gamma]$ is a cross-term. We show how various consistency conditions of the index formula require different parts of $\lambda$ to vanish.

In Sect. 2 we review the derivation of the index formula using path integrals and discuss how the $\lambda$-class appears for the Dirac-Ramond operator on a general manifold. In Sect. 3 we discuss in detail the string structure on a simply connected manifold divided by a finite abelian group of isometries. We show that the string structure can be constructed in each sector of the loop space independently of whether $\lambda$ vanishes or not. Only after one relates different sectors by requiring that the $\operatorname{Diff}^{+}\left(S^{1}\right)$ action can be lifted to the string structure, the $\lambda$-class appears as an obstruction. In Sect. 4 we explain how the index formula on an orbifold can be derived and show that part of (1.2) is required to vanish through the consistency conditions on the index formula.

\section{Index of the Dirac-Ramond Operator}

We consider the heterotic string $\sigma$-model on a Riemannian manifold $M$. Upon quantization the supersymmetry charge of the right moving sector becomes an operator on the loop space, $L M$, of $M$. This is the Dirac-Ramond operator, $F[1]$. To avoid inconsistencies in the definition of $F$ it was suggested by Killingback [14] that the manifold must possess a "string structure."

The ordinary spin structure on $M$ is associated with the central extension

$$
0 \rightarrow \mathbf{Z}_{2} \rightarrow \operatorname{Spin}(d) \rightarrow S O(d) \rightarrow 0,
$$

of the orthogonal group $S O(d)$ to $\operatorname{Spin}(d)$. Here, $S O(d)$ is the structure group of the bundle of the orthonormal frames, $P$, on $M$, and the spin bundle, $S$, if it exists, is a double cover of $P$ with the structure group $\operatorname{Spin}(d)$. As mentioned earlier the existence of the spin bundle imposes the topological restriction that $w_{2}(M)=0$. More generally, if the spinors couple to a vector bundle $E$, then the existence of the generalized spin structure requires that $w_{2}(M)-w_{2}(E)=0$.

On a loop space, $L M$, assuming that $M$ has a spin structure, the natural "frame bundle" is the bundle, $L S$, of loops in $S$, whose structure group is $L \operatorname{Spin}(d)$, i.e., the loop group of $\operatorname{Spin}(d)$. An element, $g$, of $L \operatorname{Spin}(d)$ is a smooth map $g: S^{1} \rightarrow \operatorname{Spin}(d)$. This group has a unique central extension [15], $\hat{L} \operatorname{Spin}(d)$,

$$
0 \rightarrow U(1) \rightarrow \hat{L} \operatorname{Spin}(d) \rightarrow L \operatorname{Spin}(d) \rightarrow 0,
$$

with the center $U(1)$. The string structure exists if the manifold, $M$, has a spin structure (or a generalized spin structure) and the bundle $L S$ has an extension to a bundle $\hat{L} S$ with the structure group $\hat{L} \operatorname{Spin}(d)$. In [14] it was suggested that there is a topological obstruction to the existence of $\hat{L} G$ given by the class 
$\lambda=\frac{1}{2} p_{1}(M) \in H^{4}(M, \mathbf{Z})$. More generally, if the left-moving string gauge fermions couple to a vector bundle, $E$, then the appropriate class is $\lambda=\frac{1}{2}\left(p_{1}(M)-p_{1}(E)\right)$, where $p_{1}(M)$ and $p_{1}(E)$ are the first Pontryagin class of the tangent bundle of $M$ and the vector bundle $E$, respectively. We assume here that the structure group, $G$, of $E$ is simply connected. As for the tangent space, the string structure in $E$ is defined as an extension of the loop bundle with the structure group $L G$ to a bundle with the structure group $\hat{L} G$.

One can understand the role of the $\lambda$-class in string theory as follows. In string theory one considers the space, $\operatorname{Map}(\Sigma, M)$, of maps $x: \Sigma \rightarrow M$ from the string world sheet, $\Sigma$ into a manifold, $M$, given by the coordinates $x^{\mu}\left(\sigma_{1}, \sigma_{2}\right)$. Then one tries to define the chiral determinants of the world-sheet fermions minimally coupled to the world-sheet gauge and gravitational fields induced from $M$ via map $x^{\mu}$. However, as has been discussed by many authors $[1,16-18]$, because of anomalies these determinants cannot be defined as functions on $\operatorname{Map}(\Sigma, M)$. In general, one can only construct a determinant line bundle over the space of maps $\operatorname{Map}(\Sigma, M)$. In terms of this bundle the chiral and global anomalies have a simple interpretation [17-19]: the chiral anomaly is given by the curvature of this bundle, while the global anomalies correspond to a non-trivial holonomy. To detect the local chiral anomalies, that is to measure the curvature, one must consider pairs of directions in $\operatorname{Map}(\Sigma, M)$ defining 2-parameter families of maps from $\Sigma$ into $M$. The image in $M$ of such family defines a 4-surface, and the chiral anomaly is absent if the free part of $\lambda$ restricted to this surface vanishes in cohomology. This gives the familiar condition [20] $\operatorname{Tr} F^{2}-\operatorname{Tr} R^{2}=d H$. This is once again the usual method of detecting an anomaly in $d$-dimensions by constructing the appropriate $(d+2)$ form [21]. The differences here are that the extra 2-dimensions are realized in terms of maps of $\Sigma$ into $M$, and the $(d+2)$-dimensional fields are not arbitrary, but are induced from the background fields in $M$. As a result, the anomaly restricts the background fields in $M$, and does not spell a disaster for the $\sigma$-model. Moreover, the fact that one only need to require $\operatorname{Tr} F^{2}-\operatorname{Tr} R^{2}$ to vanish in cohomology, and not require the overall coefficient of this term to vanish is merely another way of describing the anomaly cancellation mechanism in the $\sigma$-model [22].

Having cancelled the local chiral anomalies, there are still potential global anomalies corresponding to the holonomy in the phases of the determinants. These are detected by a closed path in $\operatorname{Map}(\Sigma, M)$ which corresponds to a closed 3-surface in $M$. Computation of the holonomies associated with all such 3-surfaces determines then the torsion part ${ }^{4}, \lambda^{\text {tor }}$, of $\lambda$.

At this point one might be tempted to conclude that there are anomalies unless the whole class $\lambda$ vanishes. In fact $\lambda^{\text {tor }}=0$ is a sufficient condition for the absence of global anomalies, but it may not be necessary $[18,11]$. Intuitively we may understand this as follows. Take a 3 -surface, $K$, which detects nonvanishing $\lambda^{\text {tor }}$. This will be fatal only if it is possible to arrange a 1-parameter family of string

4 This is in agreement with the universal coefficients theorem which relates the torsion part of $H^{4}(M, \mathbf{Z})$ to the torsion part of $H_{3}(M, \mathbf{Z})$, which is spanned by closed 3-surfaces in $M$ such that a finite number of them bounds a 4-submanifold in $M$ 
world-sheets to produce $K$. It may happen that the best one can do is to cover $K$ $n$-times with such a family. As a result the necessary condition will be $n \cdot \lambda^{\text {tor }}=0$. Of course, to analyze this completely is rather complicated and ultimately by considering all possible families of world-sheets we may end up with the requirement that $\lambda=0$.

To summarize, the condition for the existence of string structure of [14] might be slightly stronger than the one needed for the anomaly cancellation.

Having described the topological conditions necessary to define the DiracRamond operator coupled in a gauge and gravitational background we may now ask to what extend the index formula, analogous to (1.1), detects these conditions.

The loop space has a natural $S^{1}$-symmetry, the translation by a constant along the loop. Therefore the proper form of the index is the character valued index defined by

$$
I(\tau)=\operatorname{Tr}\left(q^{H_{L}} \bar{q}^{H_{R}}(-1)^{f_{R}}\right),
$$

where $q=e^{i \pi \tau}$, with $\operatorname{Im} \tau>0$, and $f_{R}$ is the right-moving world-sheet fermion number. The operators $H_{L}$ and $H_{R}$ are the left- and right-moving Hamiltonians, and are equal to the Virasoro generators, $L_{0}$ and $\bar{L}_{0}$, shifted by the appropriate intercept parameters, in the background fields. From the usual supersymmetry argument only the states annihilated by $H_{R}$ contribute to the trace and as a result $I(\tau)$ is always a holomorphic function. This allows one to consider (2.3) in the limit in which $\operatorname{Im} \tau$ becomes infinitesimally small, and then use analyticity to obtain the answer for arbitrary $\tau$. This was the method employed in [4]. After converting (2.3) into a path integral the limit of $\operatorname{Im} \tau$ being small corresponds to the high temperature expansion. The result is ${ }^{5}$

$$
I(\tau)=\int d x_{0}^{\mu} \int d \lambda_{0}^{\mu}\left[\frac{\operatorname{det}\left(\bar{\partial} \delta_{A B}+F_{A B}\left(x_{0}\right)\right)}{\operatorname{det}^{\prime}\left(\bar{\partial} \delta_{\sigma}^{\rho}+R_{\sigma}^{\rho}\left(x_{0}\right)\right)}\right]^{1 / 2},
$$

where $\bar{\partial}=\left(\partial_{\sigma_{1}}+\tau \hat{\partial}_{\sigma_{2}}\right)$ is the anti-holomorphic differential on a torus with Teichmüller parameter, $\tau$, and the coordinates $\sigma_{1}$ and $\sigma_{2}$ vary from 0 to $2 \pi$. The curvature 2-forms

$$
\begin{aligned}
F_{A B} & =\frac{1}{2}\left(F_{\mu \nu}\right)_{A B} \lambda_{0}^{\mu} \lambda_{0}^{v}, \\
R_{\sigma}^{\rho} & =\frac{1}{2} R_{\sigma \mu \nu}^{\rho} \lambda_{0}^{\mu} \lambda_{0}^{\nu},
\end{aligned}
$$

depend only upon the zero modes, $x_{0}$ and $\lambda_{0}$, and thus represent constant twists on the string world-sheet. The determinants in the numerator and the denominator are for free fermions and bosons, respectively, with implicit boundary conditions corresponding to the summation over world sheet spin structures. The powers of $\frac{1}{2}$ in (2.4) arise because the fields are real, and the corresponding determinants over

5 In comparison with [4] there is no overall factor $\operatorname{det}^{\prime}(\bar{\partial})$ which arises from the integration over the ghost and superghost fields. These fields do not couple to the background and can be dropped in the calculation of the index of the Dirac-Ramond operator. However, they must be included if one wants to obtain correctly space-time anomalies in the underlying string theory 
the real chiral fields are to be defined as square roots of determinants for the complex chiral fields.

The index formula $I(\tau)$ can be also obtained without path integrals by applying the fixed point theorem [23] to the Dirac-Ramond operator and the $S^{1}$-symmetry $[11,24]$. The main idea of this method is as follows ${ }^{6}$. To compute the character valued index of the Dirac operator on a manifold, $M$, with a symmetry group, $G$, it is enough to consider a Dirac operator on the submanifold, $M[G]$, which consists of fixed points of $G$-action, and couple it to the bundles which are canonically constructed from the bundle normal to the fixed point set. For the Dirac-Ramond operator on $L M$ the fixed point set is the manifold, $M$, itself, and the bundles which arise from the normal directions in the loop space are precisely those of the massive modes of the string. Therefore, the calculation of the index from the fixed point formula is nothing other than assembling the elliptic genus from the $\hat{A}$-genus and Chern characters corresponding to all mass levels of the string. This was the method, motivated by physical considerations, which was used originally in [2].

We will call $I(\tau)$ given in $(2.4)$ the index formula for the Dirac-Ramond operator. The fact that (2.4) only depends on the background fields through the curvatures localized at $x_{0}$ is a reflection of the high temperature limit in which the dominant contribution to (2.3) comes from infinitesimal tori around $x_{0}$. Integration over the non-zero modes can then be rewritten in terms of path integrals in the tangent space, and the geometry of $M$ enters only via constant twists. Therefore one would expect that the index formula will be consistent even in the presence of nontrivial geometry. However, the chiral determinants in (2.4) are infinite dimensional and must be consistently regularized over the entire manifold [3,24]. Moreover, we want to compute the character valued index of the Dirac-Ramond operator, and the path integral that corresponds to $\operatorname{Tr}(-1)^{f_{R}}$ is evaluated over tori with a distinguished 1-cycle corresponding to the $S^{1}$-action. On a euclidean torus there is no natural way to distinguish a single cycle, and this may cause an inconsistency in (2.4) unless it is independent of which way $S^{1}$ acts on a torus. This ambiguity persists even for infinitesimally small tori, and as will be seen, can detect the localizable part of $\lambda$.

As discussed in [3] the determinants in (2.4) can be holomorphically regularized and evaluated explicitly in terms of the Jacobi $\theta$-functions. The result is

$$
I(\tau)=\int d^{d} x\left[\prod_{\alpha=1}^{d / 2} \frac{x_{\alpha} \eta(\tau)}{\theta_{1}\left(x_{\alpha} / 2 \pi i \mid \tau\right)} \prod_{\beta=1}^{N} \frac{\theta_{i}\left(y_{\beta} / 2 \pi i \mid \tau\right)}{\eta(\tau)}\right]_{\mathrm{top} \mathrm{form}},
$$

where $x_{\alpha}$ and $y_{\beta}$ are 2-forms that are skew-eigenvalues of $R$ and $F$, respectively, and $\eta(\tau)$ is the Dedekind function. The particular theta-function, $\theta_{i}$, or their combination, in (2.7) depends on the boundary conditions in the left-moving sector.

The index formula in (2.2) should be viewed as a function of the torus that is used to define the determinants, and not merely as a function of $\tau$ with $\operatorname{Im} \tau>0$. As a result $I(\tau)$ is required to be a modular function for the entire modular group if one has a modular invariant string theory, or for a subgroup of the modular

6 For a detailed discussion see, e.g., $[10,11]$ 
group if one restricts to a subsector of the string. In either circumstance the transformation of $I(\tau)$ under the modular transformation $\tau \rightarrow(a \tau+b / c \tau+d)$ can be deduced $[2,3]$ from that of the $\theta$-functions

$$
\theta_{i}\left(\frac{v}{c \tau+d} \mid \frac{a \tau+b}{c \tau+d}\right)=\varepsilon e^{i \pi c v^{2} /(c \tau+d)} \sqrt{c \tau+d} \theta_{j}(v \mid \tau),
$$

where $\varepsilon$ is a phase and $j$ depends in some complicated way on $i, a, b, c$ and $d$. One finds that the integrand in (2.7) is modular invariant, up to phases of the form

$$
\exp \left[\frac{i \pi c}{c \tau+d}\left(\operatorname{Tr} F^{2}-\operatorname{Tr} R^{2}\right)\right]
$$

However, if $F$ and $R$ satisfy

$$
\operatorname{Tr} F^{2}-\operatorname{Tr} R^{2}=d H
$$

for some 3-form $H$ on $M$, then the phase factor (2.9) disappears upon integration. This is because the theta-functions in (2.7) can always be rewritten in terms of invariant polynomials in $F$ and $R[2,3]$, which are closed forms on $M$. Thus, any term involving $d H$, or powers of it, vanishes by integration by parts. As a result we see that $I(\tau)$ is only a modular function if $(2.10)$ is satisfied.

In $[1,17,18]$ it was shown that in order to define chiral determinants in a modular invariant manner, one also encounters the torsion part of the $\lambda$ class. The reason why this does not happen here is that (2.4) has been evaluated in a high temperature limit, and the minimal couplings have disappeared. The background thus only enters through curvatures. Moreover, the high temperature limit has enabled the bosonic integration to be performed. These simplifications and the invisibility of $\lambda^{\text {tor }}$ are simply consequences of the fact that we really only consider arbitrarily small tori that can be localized at $x_{0}$, and so only see the localizable part of the class.

We conclude that the index formula $I(\tau)$ is well-defined by Eq. (2.4) when the free part of the $\lambda$-class vanishes. Moreover, if $\lambda^{\text {tor }}$ and $w_{2}$ vanish, then the Dirac-Ramond operator exists, and $I(\tau)$ can be identified as its index. However, if one is on a manifold for which the only non-zero components of $\lambda$ are the torsion parts, then $I(\tau)$ is a well defined modular function, but it may not have integer coefficients. It would be interesting to investigate under what circumstances $I(\tau)$ has integer coefficients on an arbitrary manifold on which it is well-defined.

\section{String Structure on Orbifolds}

The condition, $\lambda \equiv 0$, for the existence of string structure on a general manifold, $M$, was derived in [14] using abstract techniques of algebraic topology. Such methods are not very well known to physicists and we feel that such analysis obscures the assumptions that are being made in the proof that $\lambda$ must vanish in order for there to be a string structure. Indeed, as one might expect from the experience from the spin structures, complications arise when the manifold is not simply connected. Since the majority of physical models require orbifolds or 
non-simply connected manifolds with Wilson lines, it is clearly important to examine more closely what is required for the existence of a string structure.

The first thing to observe is that if $M$ is not simply connected, the corresponding loop space, $L M$, is not a connected space and it decomposes into components corresponding to different elements in $\pi_{1}(M)^{7}$. We shall argue below that in many cases one can establish the existence of string structure in each component separately without encountering any obstruction. It is only after one tries to relate various components that one finds that the $\lambda$-class must vanish. This suggests that when $M$ is not simply connected the definition of the string structure given in [14], which was discussed in Sect. 2, must be modified.

Rather than discussing a general case, we shall study explicitly an example which is related to abelian orbifolds. Let $M$ be a connected and simply connected manifold and $\Gamma$ a discrete group acting freely on $M$. We take $\Gamma$ to be an abelian cyclic group $\mathbf{Z}_{n}$. Since the action of $\Gamma$ on $M$ is free, the space of orbits, $M_{\Gamma}=M / \Gamma$, is a manifold. Moreover, $M_{\Gamma}$ is connected, but not simply connected and $\pi_{1}\left(M_{\Gamma}\right) \equiv \Gamma$. Let $G$ be a compact Lie group and $\rho: \Gamma \rightarrow G$ an embedding of $\Gamma$ into $G$. With each embedding, $\rho$, one can associate a principal fibre bundle $P_{\rho}$ over $M_{\Gamma}$ with the structure group $G$. The total space of $P_{\rho}$ is obtained as a diagonal quotient

$$
P_{\rho}=\frac{M \times G}{\Gamma} \text {. }
$$

In (3.1) we identify $(m, g)$ with $\left(m \gamma, \rho(\gamma)^{-1} g\right), m \in M, g \in G$ and $\gamma \in \Gamma$. For convenience we take $\Gamma$ to act on $M$ from the right. The action of the structure group, $G$, on $P_{\rho}$ is defined by

$$
g_{0}:(m, g) \rightarrow\left(m, g g_{0}\right) \text {, }
$$

and obviously does not depend on which representative of the class $[(m, g)]$ is being used. Since $\Gamma$ is abelian we can always arrange the embedding, $\rho$, to be via the maximal torus, that is

$$
\begin{gathered}
\Gamma \subset T(G) \subset G \\
\rho(\gamma)=\left(\begin{array}{ccc}
e^{2 \pi i \theta_{1}} & & \\
& \ddots & \\
& & e^{2 \pi \imath \theta_{N}}
\end{array}\right),
\end{gathered}
$$

where the twists $\theta_{i}=k_{i} / n, k_{i} \in \mathbf{Z}, i=1, \ldots, N=\operatorname{rank} G$, are such that $\rho(\gamma)$ is an element of order $n$ in $G$. It has been discussed by many authors $[1,25]$ that the bundle defined in (3.1-3.3) is in general nontrivial. It is also flat and has nonvanishing Wilson lines corresponding to noncontractible loops in $M_{\Gamma}$. One can show that the Pontryagin class of $P_{\rho}$ is [25]

$$
p_{1}\left(P_{\rho}\right)=\sum_{i=1}^{N}\left(k_{i}\right)^{2} \hat{x}^{2}
$$

7 For simplicity we assume that $\pi_{1}(M)$ is an abelian group. In general one must consider loops in the conjugacy classes in $\pi_{1}(M)$ 
where $\hat{x}^{2} \in H^{4}\left(M_{\Gamma}, \mathbf{Z}\right)$ is a torsion class element such that $n \hat{x}^{2}=0$. The details of $\hat{x}^{2}$ depend on $M$. For example it vanishes if the dimension of $M_{\Gamma}$ is less than 4 . However, in a generic case, e.g., for $M=S^{2 k+1}(k \geqq 2), \hat{x}^{2}, 2 \hat{x}^{2}, \ldots,(n-1) \hat{x}^{2}$ are distinct and nonzero. For more discussion of (3.4) we refer to [1] and [25].

Before we discuss the existence of string structure on $P_{\rho}$, we consider a simpler, but conceptually similar question for the spin structure, when $G$ is an orthogonal group. If $G=S O(2 N)$ to construct a spin structure on $P_{\rho}$, one must lift the embedding $\rho$ to $\hat{\rho}: \Gamma \rightarrow \operatorname{Spin}(2 N)$. For a generator, $\gamma$, of $\Gamma, \hat{\rho}(\gamma)^{n}$ will in general be equal to \pm 1 , and one must impose that $\hat{\rho}(\gamma)$ is of order $n$ in $\operatorname{Spin}(2 N)$. When $n$ is odd such $\hat{\rho}$ always exists, but for $n$ even one must require

$$
\sum_{i=1}^{N} k_{i}=0 \bmod 2 .
$$

Having chosen the embedding $\hat{\rho}$ we can define a spin structure on $P_{\rho}$ by considering a bundle

$$
\hat{P}_{\rho}=\frac{M \times \operatorname{Spin}(2 N)}{\Gamma},
$$

which satisfies all the required conditions.

The existence of string structure in $P_{\rho}$ can be analyzed in a similar way. From now on we will assume in addition that $G$ is also simply connected and simply laced. By taking smooth loops in the total space of the bundle $P_{\rho}$ one obtains the following bundle of loop spaces

$$
\begin{aligned}
& L G \rightarrow L P_{\rho}=L \\
& \qquad M_{\Gamma}=L\left(\frac{M}{\Gamma}\right)
\end{aligned},
$$

with the structure group, $L G$, which is the group of smooth loops in $G$. Since $M$ and $G$ are connected and simply connected $\pi_{1}\left(P_{\rho}\right) \equiv \Gamma$ and the projection $\pi$ is an isomorphism between the homotopy groups. The total space $L P_{\rho}$ of the loop bundle is disconnected and $\Gamma$ labels sectors corresponding to different elements in $\pi_{1}\left(P_{\rho}\right)$. Thus for each $\gamma \in \Gamma$ we have a corresponding bundle

$$
\begin{array}{r}
L G \rightarrow L_{\gamma} P_{\rho} \\
\downarrow \\
L_{\gamma} M_{\Gamma},
\end{array}
$$

where $L_{\gamma} P_{\rho}$ and $L_{\gamma} M_{\Gamma}$ denote spaces of loops in $P_{\rho}$ and $M_{\Gamma}$ in the class $\gamma$. Since $\Gamma$ is discrete and acts freely we may lift these loops to twisted loops in $M \times G$ and $M$, respectively. Denote by $L_{\gamma} M$ and $L_{\gamma} G$ spaces of loops twisted by $\gamma$, that is

$$
\begin{gathered}
L_{\gamma} M \ni m: \mathbf{R} \rightarrow M, \quad m(t+2 \pi)=m(t) \gamma, \\
L_{\gamma} G \ni g: \mathbf{R} \rightarrow G, \quad g(t+2 \pi)=\rho(\gamma)^{-1} g(t) .
\end{gathered}
$$


We then have canonical isomorphisms

$$
\begin{aligned}
L_{\gamma}\left(\frac{M \times G}{\Gamma}\right) & =\frac{L_{\gamma} M \times L_{\gamma} G}{\Gamma}, \\
L_{\gamma} M_{\Gamma} & =\frac{L_{\gamma} M}{\Gamma}
\end{aligned}
$$

The action of $\Gamma$ on the twisted loop spaces above is the one inherited from the action on $M$ and $G$. Since $\Gamma$ is abelian, different twisted sectors do not mix. We would like to emphasise that in each twisted sector the structure group is still equal to $L G$. Indeed, if $g_{1}(t), g_{2}(t) \in L_{\gamma} G$, then $g_{1}(t)=g_{2}(t) g(t)$ for some untwisted loop $g(t)$. Thus by choosing a representative, $g_{\gamma}(t)$, in each sector we have $L_{\gamma} G=$ $g_{\gamma} \cdot L G$. This shows that there is an isomorphism

$$
L_{\gamma}\left(\frac{M \times G}{\Gamma}\right) \equiv \frac{L_{\gamma} M \times L G}{\Gamma} .
$$

However this isomorphism is not canonical since it depends on how we identify $L_{\gamma} G$ with $L G$, that is, it depends upon the base point $g_{\gamma} \in L_{\gamma} G$. In particular let $v: g(t) \rightarrow \rho_{\gamma}(v)(t) g(t)$ define the action by right multiplication of an element $v$ of $\Gamma$ on $L G$. Since $L_{\gamma} G=g_{\gamma} \cdot L G$ we must take

$$
\rho_{\gamma}(v)(t)=g_{\gamma}(t)^{-1} \rho(v)^{-1} g_{\gamma}(t)
$$

Observe that $\rho_{\gamma}$ defines an embedding of $\Gamma$ into $L G$, and thus the structure of the bundle in (3.13) is exactly the same as of the one in (3.1).

To define a string structure we will show that one can define a lift of $\rho_{\gamma}$ to the central extension $\hat{L} G$. Here $\hat{L} G$ is the basic central extension [15] of $L G$. The corresponding exact sequence is

$$
0 \rightarrow U(1) \rightarrow \widehat{L} G \rightarrow L G \rightarrow 0 .
$$

As a manifold $\hat{L} G$ is a $U(1)$ bundle over $L G$. The first Chern class of this bundle is given by a left-invariant 2 -form, $(1 / 2 \pi) \omega$, on $L G$. At the origin $\omega$ is a cocycle on the Lie algebra of $L G$ defined by

$$
\omega(\xi, \eta)=\frac{1}{2 \pi} \int_{0}^{2 \pi} d t\left\langle\xi(t), \eta^{\prime}(t)\right\rangle,
$$

where $\xi(t)$ and $\eta(t)$ are loops in the Lie algebra, $\mathscr{G}$, of $G$, and $\langle$,$\rangle is the basic$ invariant product in $\mathscr{G}$, normalized so that the length of roots is equal to $\sqrt{2}$. This extension is basic in the sense that any other can be obtained from it by dividing out a normal subgroup.

There is a convenient method of describing $\hat{L} G$ more explicitly [15]. One considers the set of triples $[g(t), p(g), u]$, where $g(t) \in L G, p(g)$ is a path in $L G$ from the unit element to $g(t)$, and $u \in U(1)$. In this set one can define an equivalence relation such that $[g(t), p(g), u] \equiv\left[g^{\prime}(t), p^{\prime}\left(g^{\prime}\right), u^{\prime}\right]$ if and only if $g(t)=g^{\prime}(t)$ and $u=C\left(p^{\prime} * p^{-1}\right) u^{\prime}$. Here $p^{\prime} * p^{-1}$ denotes a closed path in $L G$ obtained by going first 
along $p^{\prime}$ and then back along $p$, and

$$
C\left(p^{\prime} * p^{-1}\right)=e^{i \int_{\sigma} \omega}
$$

where $\sigma$ is an arbitrary surface in $L G$ bounded by $p^{\prime} * p^{-1}$. Since $(1 / 2 \pi) \omega$ is a Chern class of a bundle, it is an integer class in the cohomology of $L G$ and (3.17) does not depend on which surface $\sigma$ is being used to evaluate it. As a set $\hat{L} G$ is then the set of all triples divided by the relation we have just discussed. The group multiplication in $\hat{L} G$ is

$$
\left[g_{1}, p_{1}, u_{1}\right] \cdot\left[g_{2}, p_{2}, u_{2}\right]=\left[g_{1} g_{2}, p_{1} * g_{1} p_{2}, u_{1} u_{2}\right] .
$$

It is straightforward to verify that this is well defined with respect to the equivalence relation. The projection on the first entry in $[,$,$] is the homomorphism onto L G$ whose kernel is the center $U(1)$.

Consider now the embedding $\rho_{\gamma}$. To construct its lift $\hat{\rho}_{\gamma}: \Gamma \rightarrow \hat{L} G$ it is sufficient to determine it on the generator $v \in \Gamma$ so that $\left[\hat{\rho}_{\gamma}(v)\right]^{n}=1$. If $\hat{v}$ is any element in $\hat{L} G$ that projects onto $\rho_{\gamma}(v)$ then $\hat{v}^{n}$ lies in the center of $\hat{L} G$. We can take $\hat{\rho}_{\gamma}(v)=\left(\hat{v}^{n}\right)^{-1 / n} \hat{v}$. Having constructed an embedding $\hat{\rho}_{\gamma}$ in each sector one merely observes that the bundle

$$
\hat{L} P_{\rho}=\bigcup_{\gamma \in \Gamma} \frac{L_{\gamma} M \times \hat{L} G}{\Gamma}
$$

defines a string structure in $P_{\rho}$. Note that this construction is completely independent on whether the $\lambda$-class vanishes or not.

In the above construction of the string structure all the twisted sectors were treated independently. The string structure was obtained by lifting separately each $\rho_{\gamma}$ to $\hat{\rho}_{\gamma}$. However we must still understand whether this is sufficient to define the Dirac-Ramond operator and calculate its index. In Sect. 2 we discussed that the circular symmetry on the loop space, $L M$, enables one to reduce the index computation to the infinitesimal neighbourhood of the fixed point set, that is of the manifold, $M \subset L M$. The use of the fixed point formula is legitimate provided we can show that $S^{1}$ is indeed a symmetry of the operator. Even before we raise this question, we must understand how the symmetry group acts on the bundle to which the Dirac-Ramond operator is coupled. Therefore in the remainder of this section we investigate the conditions under which the string structure has the necessary $S^{1}$ symmetry.

On the Hilbert space of the string the generator of the $S^{1}$ action is the operator $L_{0}-\bar{L}_{0}$, where $L_{0}$ and $\bar{L}_{0}$ are the generators in the Virasoro algebra of the left-movers and the right-movers, respectively. Requiring that the states are invariant under a complete rotation around the $S^{1}$ restricts the values of the intercept parameters in $L_{0}$ and $\bar{L}_{0}$. This is the so-called level matching condition $[26,27]$. However, for a given manifold the values of the intercept parameters are fixed and can be calculated directly (for example by the $\zeta$-function regularization [26]). Thus the level matching condition is an obstruction to having a consistent string theory, and this is how the $\lambda$-class emerges for strings on orbifolds [27,28].

The $S^{1}$ group acting on the loop space is a subgroup of a larger group, namely 
$\operatorname{Diff}^{+}\left(S^{1}\right)$. In string theory one usually finds that once one represents the action of this $S^{1}$ on the Hilbert space of states one can automatically represent the whole $\operatorname{Diff}^{+}\left(S^{1}\right)$. More precisely, the action of $S^{1}$ is always represented by an operator with an intercept that can be determined in a number of ways (e.g. by $\zeta$-function regularization). The important thing is that when one constructs the other Virasoro generators from the oscillators one finds that the central term takes its canonical form when one chooses the proper value of the intercept parameter. Thus from the point of view of representation theory the correct representation of the $S^{1}$ action can only be determined by appealing to the whole of $\operatorname{Diff}^{+}\left(S^{1}\right)$ in order to determine the value of the intercept.

Consider the total space of the loop bundle $L_{\gamma} P_{\rho}$. The points of this space are loops $p: \mathbf{R} \rightarrow P_{\rho}, p(t+2 \pi)=p(t)$, where $p$ belongs to the homotopy class labelled by $\gamma$. On this space of loops there is a natural action of the group $\operatorname{Diff}^{+}\left(S^{1}\right)$. The elements of $\operatorname{Diff}^{+}\left(S^{1}\right)$, or more precisely of its universal covering, $\overline{\operatorname{Diff}^{+}\left(S^{1}\right)}$, are mappings

$$
\varphi: \mathbf{R} \rightarrow \mathbf{R} \quad \varphi(t+2 \pi)=\varphi(t)+2 \pi,
$$

and the action on $p(t)$ is given by

$$
\varphi \cdot p(t)=p\left(\varphi^{-1}(t)\right)
$$

Note that this action is an automorphism of the bundle in the sense that

$$
\varphi \cdot(p g)=(\varphi \cdot p)(\varphi \cdot g)
$$

where $g \in L G$ and $(\varphi \cdot g)(t)=g\left(\varphi^{-1}(t)\right)$ is the natural action of $\overline{\operatorname{Diff}^{+}\left(S^{1}\right)}$ on $L G$.

If we choose to describe $L_{\gamma} P_{\rho}$ in terms of the twisted loop space $\left(L_{\gamma} M \times L G\right) / \Gamma$ as in (3.13), we find that a pair $(m(t), g(t))$ transforms under $\varphi$ as follows

$$
\begin{aligned}
\varphi: m(t) & \rightarrow m\left(\varphi^{-1}(t)\right), \\
\varphi: g(t) \rightarrow R_{\gamma}[\varphi](t)(\varphi \cdot g)(t) & =g_{\gamma}^{-1}(t) g_{\gamma}\left(\varphi^{-1}(t)\right) g\left(\varphi^{-1}(t)\right) .
\end{aligned}
$$

One must multiply $\varphi \cdot g(t)$ by $R_{\gamma}[\varphi](t)=g_{\gamma}^{-1}(t) g_{\gamma}\left(\varphi^{-1}(t)\right)$ because the base point, $g_{\gamma}(t)$, gets transformed under $\varphi$. Also, one should notice that it is really a $k$-fold cover of $\operatorname{Diff}^{+}\left(S^{1}\right)$ (where $k$ is the order of $\gamma$ in $\Gamma$ ) rather then $\operatorname{Diff}^{+}\left(S^{1}\right)$ that acts on $L_{\gamma} M \times L G$. For later purposes we introduce a notation for the diffeomorphisms which correspond to the $\mathbf{R}$ subgroup of $\overline{\operatorname{Diff}^{+}\left(S^{1}\right)^{8}}, \varphi_{\alpha}(t)=t-\alpha$. From (3.24) we find that the translation by $2 \pi$ in $L G$ gives the embedding $\rho_{\gamma}(\gamma)(t)=R_{\gamma}\left[\varphi_{2 \pi}\right](t)$.

Consider the untwisted sector. We want to check that the action of Diff ${ }^{+}\left(S^{1}\right)$ on the bundle $L_{0} P_{\rho}$ can be lifted to an automorphism of the untwisted sector of the bundle (3.19). First we shall describe how the $\operatorname{Diff}^{+}\left(S^{1}\right)$ action lifts from $L G$ to $\hat{L} G$. Such a lift exists and is unique [15]. On the explicit realization of $L G$, which was discussed above, this action can be described as follows

$$
\varphi:[g(t), p(g), u] \rightarrow \hat{\varphi} \cdot[g(t), p(g), u]=[(\varphi \cdot g)(t), \varphi \cdot p(g), u],
$$

8 This corresponds to the $S^{1}$ subgroup of $\operatorname{Diff}^{+}\left(S^{1}\right)$ 
where $\varphi \cdot p(g)$ is a path obtained by transforming with $\varphi$ all the points on the path $p(g)$. Since the cocycle (3.16) is manifestly invariant under reparametrizations the transformation defined in (3.25) does not depend on a choice of a representative of a given element in $\hat{L} G$. This action is also an automorphism of $\hat{L} G$, that is

$$
\hat{\varphi} \cdot\left(\hat{g}_{1} \hat{g}_{2}\right)=\left(\hat{\varphi} \cdot \hat{g}_{1}\right)\left(\hat{\varphi} \cdot \hat{g}_{2}\right) \quad \hat{g}_{1}, \hat{g}_{2} \in \hat{L} G .
$$

The elements of $\hat{L} G$ that project onto constant loops in $L G$ are inert under this action of $\operatorname{Diff}^{+}\left(S^{1}\right)$. In particular the center $U(1)$ is inert.

In the untwisted sector the embedding $\rho_{0}: \Gamma \rightarrow G \subset L G$ takes $\Gamma$ to constant loops. Thus $\rho_{0}(\Gamma) \subset \hat{L} G$ does not transform under $\operatorname{Diff}^{+}\left(S^{1}\right)$. The action of $\operatorname{Diff}^{+}\left(S^{1}\right)$ on $L M \times \hat{L} G$ factors to the quotient $\hat{L}_{0} P_{\rho}$ and defines the lift of the action on $L_{0} P_{\rho}$ we are looking for.

The twisted sector is more complicated to analyze. Because of the base point we must use the modified action of $\operatorname{Diff}^{+}\left(S^{1}\right)$ on $L G$ given in (3.24) and it is this that must be lifted to $\hat{L} G$. Denote this lift by $\hat{T}$. To obtain an automorphism of the string structure of the form (3.22) $\hat{T}$ must satisfy

$$
\hat{T}_{\varphi}\left(\hat{g}_{1} \hat{g}_{2}\right)=\hat{T}_{\varphi}\left(\hat{g}_{1}\right)\left(\hat{\varphi} \cdot \hat{g}_{2}\right) \quad \hat{g}_{1}, \hat{g}_{2} \in \hat{L} G .
$$

Thus it is sufficient to determine $\hat{T}_{\varphi}$ on the unit element $\hat{\mathbf{1}}$ of $\hat{L} G$. Denote

$$
\hat{T}_{\varphi}(\hat{\mathbf{1}})=\hat{R}_{\gamma}(\varphi) .
$$

The projection of $\hat{R}_{\gamma}(\varphi)$ on $L G$ is equal to $R_{\gamma}(\varphi)$ given in (3.24). From (3.27) we deduce that it must satisfy the following consistency conditions

$$
\begin{gathered}
\hat{R}_{\gamma}(i d)=\hat{\mathbf{1}}, \\
\hat{R}_{\gamma}(\psi \circ \varphi)=\hat{R}_{\gamma}(\psi) \hat{\psi} \cdot \hat{R}_{\gamma}(\varphi) .
\end{gathered}
$$

These conditions guarantee that $\hat{T}$ defines a group action. Conditions similar to (3.29) and (3.30), but without the "hats," are also satisfied by $R_{\gamma}$.

Similarly as in the untwisted case we can show that if the action of $\operatorname{Diff}^{+}\left(S^{1}\right)$ on $L G$, defined by (3.24), lifts to an action on $\hat{L} G$ then this lift is unique. Indeed, let $\hat{R}_{\gamma}$ and $\hat{R}_{\gamma}^{\prime}$ correspond to two different lifts. Then $\Delta_{\gamma}(\varphi)=\hat{R}_{\gamma}(\varphi) \hat{R}_{\gamma}^{\prime}(\varphi)^{-1}$ is a mapping from $\overline{\operatorname{Diff}^{+}\left(S^{1}\right)}$ to the center $U(1)$. Using (3.26), (3.29), (3.30) and the triviality of $\hat{\varphi}$ on the center we find that $\Delta$ is in fact a group homomorphism and thus must be trivial.

Observe that $R_{\gamma}$ defines a mapping

$$
R_{\gamma}: \overline{\operatorname{Diff}^{+}\left(S^{1}\right)} \rightarrow L G
$$

The 2-form $\omega$ on $L G$, which defines the central extension, can be pulled back by $R_{\gamma}$ onto $\overline{\operatorname{Diff}^{+}\left(S^{1}\right)}$. Equation (3.30) (without the hats) and the invariance of $\omega$ under the left-multiplication in $L G$ and under $\operatorname{Diff}^{+}\left(S^{1}\right)$ imply that $R_{\gamma}^{*} \omega$ is a left-invariant form on $\overline{\operatorname{Diff}^{+}\left(S^{1}\right)}$. Thus it is sufficient to determine it at the origin, that is on the generators, $L_{m}, m \in \mathbf{Z}$, of the Virasoro algebra. In the complexified tangent space we have

$$
L_{m}=i e^{i m t} \frac{d}{d t}
$$


From (3.16) and (3.24) we then find

$$
R_{\gamma}^{*} \omega\left(L_{m}, L_{n}\right)=\frac{1}{4 \pi} i(m-n) \int_{0}^{2 \pi} e^{i(m+n) t} \Lambda(t) d t=\frac{1}{2} i(m-n) \Lambda_{m+n},
$$

where

$$
\Lambda(t)=\left\langle g_{\gamma}(t)^{-1} \frac{d}{d t} g_{\gamma}(t), g_{\gamma}(t)^{-1} \frac{d}{d t} g_{\gamma}(t)\right\rangle
$$

depends only on the twisted loop $g_{\gamma}(t)$. From the cohomology of $\operatorname{Diff}^{+}\left(S^{1}\right)$ it is known that the form in (3.33) is trivial. In fact

$$
R_{\gamma}^{*} \omega=d A,
$$

where $A$ is a left invariant form on $\overline{\operatorname{Diff}^{+}\left(S^{1}\right)}$. At the origin $A$ is given by

$$
A\left(L_{m}\right)=-\frac{1}{2} i \Lambda_{m}
$$

To proceed further we will choose an explicit twisted loop

$$
g_{\gamma}(t)=\left(\begin{array}{lll}
e^{i\left(k_{1} / n\right) t} & & \\
& \ddots & \\
& & e^{i\left(k_{N} / n\right) t}
\end{array}\right),
$$

which lies in the maximal torus. The product $\langle$, $\rangle$ in (3.33) restricted to the Cartan algebra is equal to - Tr. From (3.33) and (3.35) we then find

$$
R_{\gamma}^{*} \omega\left(L_{m}, L_{n}\right)=i m\left[\sum_{i=1}^{N}\left(\theta_{i}\right)^{2}\right] \delta_{m+n, 0},
$$

and

$$
A\left(L_{m}\right)=-\frac{1}{2} i\left[\sum_{i=1}^{N}\left(\theta_{i}\right)^{2}\right] \delta_{m, 0} .
$$

To construct $\hat{R}_{\gamma}(\varphi)$, and thereby obtain $\hat{T}_{\varphi}$, we will modify $\hat{\varphi}$ given in (3.25). The mapping, $R_{\gamma}$, in an neighbourhood, $\mathscr{U}$, of the unit element, is an injection. Moreover it will be important later to observe that we can choose $\mathscr{U}$ to include the identity of $\operatorname{Diff}^{+}\left(S^{1}\right)$ as well as $\varphi_{2 \pi}$. (This follows from the fact that if $R_{\gamma}(\varphi)=R_{\gamma}\left(\varphi^{\prime}\right)$, then $\left.\left(k_{i} / n\right)\left(\varphi^{-1}(t)-\varphi^{\prime-1}(t)\right)=2 \pi l_{i}, l_{i} \in \mathbf{Z}, i=1, \ldots, N\right)$. If $\varphi \in \mathscr{U}$ then define

$$
\hat{R}_{\gamma}(\varphi)=\left[R_{\gamma}(\varphi), R_{\gamma}\left(p_{\varphi}\right), \Phi\left(p_{\varphi}\right)\right],
$$

where $p_{\varphi}$ is a path in $\mathscr{U}$ from the identity to $\varphi$, and $R_{\gamma}\left(p_{\varphi}\right)$ is its image in $L G$. The phase $\Phi\left(p_{\varphi}\right)$ is obtained by integrating the potential $A$ along $p_{\varphi}$,

$$
\Phi\left(p_{\varphi}\right)=e^{-i \int_{p \varphi} A} .
$$

One can check that $\hat{R}_{\gamma}(\varphi)$ defined in (3.40) does not depend on the choice of the path $p_{\varphi}$, as long as the latter is in $\mathscr{U}$. The last restriction is necessary so that the phase (3.17) can be computed by pulling back the integral over 2-surface to 
$\overline{\operatorname{Diff}^{+}\left(S^{1}\right)}$ and replacing it by a line integral of the potential $A$ along the boundary formed by two paths one is comparing. Using the left-invariance of $A$ one can also show that, when $\varphi, \psi, \psi \circ \varphi \in \mathscr{U}, \hat{R}_{\gamma}$ satisfies the consistency conditions (3.29) and (3.30).

The last thing we must check is that $\left(\widehat{T}_{\varphi_{2 \pi}}\right)^{n}=$ id. Since $\hat{\varphi}_{2 \pi}$ defined by (3.25) is the identity map, all we need to show is that $\left[\hat{R}_{\gamma}\left(\varphi_{2 \pi}\right)\right]^{n}=\hat{\mathbf{1}}$ in $\hat{L} G$. If we choose the path $p(\tau), \tau \in[0,1]$ in (3.40) as $p\left[\varphi_{2 \pi}\right](\tau)=\varphi_{2 \pi \tau}$, then its image by $R_{\gamma}$ will be a path in $G \subset L G$. Using (3.32) we find that $\varphi_{2 \pi \tau}$ can also be written as $\varphi_{2 \pi \tau}=$ $\exp \left(2 \pi i \tau L_{0}\right)$. Comparing with (3.39) we see that the line integral of $A$ along $p$ becomes straightforward. The resulting phase (3.41) is

$$
\Phi(p)=\exp \left(-\pi i \sum_{i=1}^{N}\left(\theta_{i}\right)^{2}\right) .
$$

Putting everything together we obtain

$$
\left[\hat{R}_{\gamma}\left(\varphi_{2 \pi}\right)\right]^{n}=\left[\rho(\gamma)^{n}, r * \rho(\gamma) \cdot r * \cdots * \rho(\gamma)^{n-1} \cdot r, \Phi(p)^{n}\right],
$$

where $r=R_{\gamma}\left(p\left[\varphi_{2 \pi}\right]\right)$, that is $r(\tau)=g_{\gamma}(2 \pi \tau)$. The first entry on the right-hand side is $\mathbf{1}$ since $[\rho(\gamma)]^{n}=\mathbf{1}$. The second entry is a closed path of constant loops, that is it lies entirely in $G \subset L G$, and so is equivalent to a constant path at $\mathbf{1}$ under the equivalence relation on such triples, provided one introduces the phase dictated by (3.17). However $\omega$ vanishes on $G \subset L G$, and so this phase is trivial. Thus (3.43) is equal to $\hat{\mathbf{1}}$ if and only if $\Phi(p)^{n}=1$, that is

$$
\frac{1}{2} \sum_{i=1}^{N}\left(k_{i}\right)^{2}=0 \bmod n
$$

This is the level matching condition of ref. [26,27]. Comparing with (3.4) we see that (3.44) is exactly the condition for the vanishing of the $\lambda$-class [28].

The obstruction to the existence of a string structure must be a topological invariant, and from (3.44) we see that the relevant invariant is half of the first Pontryagin class of the bundle $P_{\rho}$. Thus, if instead of the twisted loop (3.37) we used any other twisted loop which could be obtained by a continuous deformation, the result would be still the same. Since $G$ is connected and simply connected, $L G$ is connected and the derivation of the obstruction is thus independent of the choice of $g_{\gamma}$. It is clear that the choice (3.37) of the twisted loop significantly simplified the derivation of (3.44). It would be still interesting to derive this result without explicit calculations.

Throughout this section we have been assuming that $\Gamma$ acts on $M$ freely. This is obviously not the case on orbifolds. However, as has been discussed in detail in [28], one should then use the equivariant construction [29], and replace $M$ with a larger space, which is homotopically equivalent to $M$ and on which $\Gamma$ acts freely. Since the existence of the string structure and the index problem for the Dirac-Ramond operator do not depend on continuous deformations, we can use this construction to generalize our results to orbifolds.

Let $M$ be an arbitrary manifold. When the action of $\Gamma$ has fixed points, $M / \Gamma$ is an orbifold and the proper generalization of $M_{\Gamma}$ is the equivariant quotient 
defined as the manifold $M_{\Gamma}=\left(M \times E_{\Gamma}\right) / \Gamma$, where $E_{\Gamma}$ is some contractible space on which $\Gamma$ acts freely. In our case one can take $E_{\Gamma}$ to be a unit sphere, $S^{\infty}$, in an infinite dimensional complex Hilbert space, i.e., $S^{\infty}=\left\{\left.\left(z_{1}, z_{2}, \ldots,\right)\left|\sum_{i=1}^{\infty}\right| z_{i}\right|^{2}=1\right.$, $\left.z_{i} \in \mathbf{C}\right\}$. The action of $[k] \in \Gamma$ on $S^{\infty}$ is $[k]\left(z_{1}, \ldots\right)=\left(e^{2 \pi i k / n} z_{1}, \ldots\right)$. The quotient $B_{\mathrm{Z}_{n}}=S^{\infty} / \Gamma$ is called a classifying space for $\mathbf{Z}_{n}$.

By replacing everywhere $M$ with $M \times E_{\Gamma}$ we find (3.44) as the obstruction to the existence of string structure on an abelian flat orbifold.

In the most general situation we may consider $M$ together with nontrivial gauge and gravitational backgrounds on which $\Gamma$ acts as a symmetry group. In this case the $\lambda$ class of the quotient bundles will have contribution from the twists and from the backgrounds. The full $\lambda$ class is then given by the equivariant Pontryagin class [28]. For the tangent bundle on $M$ it is defined as

$$
p_{1}(M / \Gamma)=p_{1}\left(M_{\Gamma}\right) \in H^{4}\left(M_{\Gamma}, \mathbf{Z}\right) .
$$

When restricted to a connected component of the subspace of the fixed points, $M[\Gamma]$, in $M$, it can be decomposed into the cohomology classes of $M[\Gamma]$ and $B_{\Gamma}$. On $M[\Gamma]$ the tangent space $T M$ can be decomposed into

$$
T M=T M[\Gamma] \oplus\left[\bigoplus_{i} T^{(i)}\right],
$$

where $T M[\Gamma]$ is the component tangent to $M[\Gamma]$, and $T^{(i)}$ are $d_{i}$-dimensional complex bundles, in the normal direction, on which $\Gamma$ acts by a multiplication with $e^{2 \pi i \theta_{i}}, \theta_{i}=k_{i} / n^{9}$. Let $\mathscr{L}$ be a line bundle over $B_{\mathbf{z}_{n}}$ defined as a quotient $\mathscr{L}=\left(S^{\infty} \times \mathbf{C}\right) / \Gamma$, where on $\mathbf{C},[k] z=e^{2 \pi i k / n} z$. The decomposition of $p_{1}(M / \Gamma)$, restricted on $M[\Gamma]$ is obtained by a straightforward computation in cohomology from the decomposition (3.46). We will not describe it here. It can be found, for example, in [12]. The result is

$$
\left.\frac{1}{2} p_{1}(M / \Gamma)\right|_{M[\Gamma]}=\frac{1}{2} p_{1}\left(\left.T M\right|_{M[\Gamma]}\right)+\frac{1}{2} \sum_{i}\left[k_{i} c_{1}\left(T^{(i)}\right) c_{1}(\mathscr{L})-\left(k_{i}\right)^{2} d_{i} c_{1}(\mathscr{L})^{2}\right],
$$

where $c_{1}\left(T^{(i)}\right)$ and $c_{1}(\mathscr{L})$ are the first Chern classes of $T^{(i)}$ and $\mathscr{L}$, respectively. Note that $T^{(i)}$ and $\mathscr{L}$ are bundles over $M[\Gamma]$ and $B_{\Gamma}$, respectively. In two extreme cases, when $\Gamma$ acts trivially and when it has only isolated fixed points, we recover the usual Pontryagin class of the manifold itself, or of the flat orbifold. Since $n c_{1}(\mathscr{L})^{2}=0$, in the latter case one recovers the level matching conditions (3.44). The decomposition (3.47) is a rigorous form of Eqs. (1.2). In the next section we will study how the equivariant $\lambda$ class (3.47) can be seen by the index formula for the Dirac-Ramond operator.

\footnotetext{
9 In general there may be also real factors in the sum (3.46). It is possible to include them but it complicates the picture significantly without providing any new insight
} 


\section{Index Formula on Orbifolds}

We consider an arbitrary manifold, $M$, with an isometry group, $\Gamma$, that also is a symmetry of the Yang-Mills background. We assume that $\Gamma$ is abelian, though for most of the discussion it can be an arbitrary discrete group. The action of $\Gamma$ on $M$ may have fixed points, so $M / \Gamma$ is an orbifold. The index formula for the Dirac-Ramond operator on $M / \Gamma$ can be obtained by generalizing (2.3) to include contributions from the twisted sectors [30]. From the point of view of the loop space, $L(M / \Gamma)$ this means including twisted loops in $M$, and also considering $\Gamma \times S^{1}$ rather than $S^{1}$ character valued indices. The twisted character valued index is defined by

$$
I\left(g_{1}, g_{2} ; \tau\right)=\operatorname{Tr}_{g_{2}}\left[q^{H_{L}} \bar{q}^{H_{R}} g_{1}(-1)^{f_{R}}\right],
$$

where $g_{1}, g_{2} \in \Gamma$ are the twists in the $\sigma_{1}$ and $\sigma_{2}$ directions, respectively. Consistency of the boundary conditions requires that $g_{1}$ and $g_{2}$ commute. As in Sect. 2 the trace in the left-moving sector is over either the Ramond or Neveu-Schwarz states and might be G.S.O. projected, depending on a particular theory.

Since the bosons and fermions in the right-moving sector are twisted in the same way, the world-sheet supersymmetry is unbroken and one can evaluate (4.1) using the usual path integral argument leading to the fixed point formula for the Dirac-Ramond operator. As a result one obtains an expression for $I\left(g_{1}, g_{2} ; \tau\right)$ in terms of an integral of various characteristic classes over the submanifold, $M_{g_{1} g_{2}}$, of points in $M$ that are fixed under the action of both $g_{1}$ and $g_{2}$. Details of this derivation can be found in [30], and here we only state the final result.

Let $V$ be a real vector bundle over $M$ to which the action of $\Gamma$ lifts. In our case $V$ is either the tangent bundle, $T M$, or the gauge bundle, $E$, to which the left-moving sector couples. Under the action of $g_{1}$ and $g_{2}, V$, restricted to $M_{g_{1} g_{2}}$, formally decomposes as follows

$$
\left.V\right|_{M_{g_{1} g_{2}}}=V^{(0)} \oplus\left[\bigoplus_{r} V^{(r)}\right] .
$$

In this decomposition $V^{(0)}$ is the part of the bundle inert under the action of $g_{1}$ and $g_{2}$. The second term in (4.2) represents a formal sum of 2-dimensional (real) bundles on which $g_{1}$ and $g_{2}$ act as rotations by the angles $2 \pi \xi_{r}$ and $2 \pi \zeta_{r}$, respectively. If $V$ is the tangent bundle, $T M,(4.2)$ corresponds to the decomposition (3.46) considered before, with $V^{(0)}=T M_{g_{1} g_{2}}$, and $V^{(i)}=T^{(i)}$. In this case the twist angles will be denoted by $2 \pi \alpha_{i}$ and $2 \pi \beta_{i}, i=1, \ldots, d_{n} / 2$, where $d_{n}$ is the dimension of the normal bundle to $M_{g_{1} g_{2}}{ }^{10}$.

Let $\lambda_{0}^{a}, a=1, \ldots, d_{p}=\operatorname{dim}\left(M_{g_{1} g_{2}}\right)$ be the zero modes of the right-moving fermions with indices tangent to $M_{g_{1} g_{2}}$. Since the gauge and gravitational backgrounds are symmetric under $\Gamma$, we can choose bases in the fibers of $E$ and $T M$ such that $F$ and $R$, with the space-time indices projected onto $M_{g_{1} g_{2}}$, have a block-

\footnotetext{
${ }^{10}$ If dimension of $M_{g_{1} g_{2}}$ is odd, the index is identically zero. Thus we need only to consider the case when $d_{n}$ is even
} 
diagonal form which coincides with the decomposition (4.2). In particular, on $T^{(i)}$ and $E^{(r)}$ we have

$$
\begin{aligned}
& \frac{1}{2} R_{a b}^{(i)} \lambda_{0}^{a} \lambda_{0}^{b}=\left(\begin{array}{rr} 
& \omega_{i} \\
-\omega_{i} &
\end{array}\right) \quad i=1, \ldots, d_{n} / 2, \\
& \frac{1}{2} F_{a b}^{(r)} \lambda_{0}^{a} \lambda_{0}^{b}=\left(\begin{array}{ll} 
& \rho_{r} \\
-\rho_{r} &
\end{array}\right) \quad r=1, \ldots, d_{n}^{E} / 2 .
\end{aligned}
$$

The character valued index (4.1) is given by the following expression

$$
\begin{aligned}
I\left(g_{1}, g_{2} ; \tau\right)= & \int_{M_{q_{1} g_{2}}} d x_{0}^{a} \int d \lambda_{0}^{a}\left[\frac{\operatorname{det}\left(\bar{\partial} \delta_{A B}+F_{A B}\right)}{\operatorname{det}^{\prime}\left(\bar{\partial} \delta_{b}^{a}+R_{b}^{a}\right)}\right]^{1 / 2} \\
& {\left[\prod_{\frac{\prod_{n}=1}{d_{n}^{E} / 2} \operatorname{det}_{\xi_{r}, \zeta_{r}}\left(\bar{\partial}+i \rho_{r}\right) \operatorname{det}_{-\xi_{r},-\zeta_{r}}\left(\bar{\partial}-i \rho_{r}\right)}^{\prod_{i=1}^{d_{n}} \operatorname{det}_{\alpha_{i}, \beta_{i}}\left(\bar{\partial}+i \omega_{i}\right) \operatorname{det}_{-\alpha_{i},-\beta_{i}}\left(\bar{\partial}-i \omega_{i}\right)}\right]^{1 / 2} . }
\end{aligned}
$$

The first part of the integrand in (4.5) is the same as in (2.4), but with $F$ and $R$ projected onto $M_{g_{1} g_{2}}$ and the tangent space and gauge indices restricted to the sectors that are inert under $g_{1}$ and $g_{2}$. In the denominator, $\operatorname{det}_{\alpha, \beta}(\bar{\partial}+v)$ denotes the determinant of $\bar{\partial}+v$ for complex modes $\psi\left(\sigma_{1}, \sigma_{2}\right)$ with boundary conditions

$$
\begin{aligned}
& \psi\left(\sigma_{1}+2 \pi, \sigma_{2}\right)=e^{2 \pi i \alpha} \psi\left(\sigma_{1}, \sigma_{2}\right), \\
& \psi\left(\sigma_{1}, \sigma_{2}+2 \pi\right)=e^{2 \pi i \beta} \psi\left(\sigma_{1}, \sigma_{2}\right) .
\end{aligned}
$$

In the numerator the boundary conditions are essentially the same except that there will be extra " - " signs depending on whether the trace involves $(-1)^{f_{L}}$, and whether the left moving fermions are in the Neveu-Schwarz or Ramond sectors. More precisely, $\operatorname{det}_{\xi, \zeta}(\bar{\partial}+v)$ is interpreted as in Sect. 2, but with the boundary conditions given an additional twist of $e^{2 \pi i \xi}$ and $e^{2 \pi i \zeta}$. As before the quantities $R_{b}^{a}, F_{A B}, \rho_{r}$ and $\omega_{i}$ are independent of $\sigma_{1}$ and $\sigma_{2}$, and are thus simply constant insertions as far as the determinants are concerned. However, these quantities do depend upon $x_{0}^{a}$.

If there are no fixed points then $I\left(g_{1}, g_{2} ; \tau\right)$ vanishes, and if $M_{g_{1} g_{2}}$ consists of isolated points then $I\left(g_{1}, g_{2} ; \tau\right)$ essentially reduces to the usual orbifold partition function given by the second part of (4.5), with no constant insertions, and summed over the fixed points.

The problem of global anomalies in (4.5) can be studied as in Sect. 2 by rewriting $I\left(g_{1}, g_{2} ; \tau\right)$ in terms of theta-functions. To each twisted determinant, with $v=0$, we associate the following function [31]

$$
\begin{aligned}
\operatorname{det}_{\alpha, \beta}(\bar{\partial}) & =d\left[\begin{array}{l}
\alpha \\
\beta
\end{array}\right](0 \mid \tau) \\
& =e^{i \pi(\alpha \beta-\beta)} q^{\left(\beta^{2}-\beta+1 / 6\right) / 2} \prod_{n=1}^{\infty}\left(1-q^{n-\beta} e^{2 \pi i \alpha}\right)\left(1-q^{n+\beta-1} e^{-2 \pi i \alpha}\right) .
\end{aligned}
$$

The overall phase in (4.8) is arbitrary and we must show that it can be chosen in 
a consistent manner. (For later comparison we use here the conventions of [27].) As discussed in [3], one can evaluate $\operatorname{det}_{\alpha, \beta}(\bar{\partial}+v)$ by absorbing the constant shift as a twist in the boundary conditions, that is by letting $\alpha \rightarrow \alpha+v$ and $\beta \rightarrow \beta$, and obtain

$$
\operatorname{det}_{\alpha, \beta}(\bar{\partial}+v)=d\left[\begin{array}{c}
\alpha \\
\beta
\end{array}\right](v \mid \tau)=e^{-i \pi \beta v} d\left[\begin{array}{c}
\alpha+v \\
\beta
\end{array}\right](0 \mid \tau)
$$

The phase in (4.9) is chosen such that $d\left[\begin{array}{l}\alpha \\ \beta\end{array}\right](v \mid \tau)$ satisfies

$$
\begin{aligned}
& d\left[\begin{array}{c}
\alpha+1 \\
\beta
\end{array}\right](v \mid \tau)=-e^{-i \pi \beta} d\left[\begin{array}{l}
\alpha \\
\beta
\end{array}\right](v \mid \tau), \\
& d\left[\begin{array}{c}
\alpha \\
\beta+1
\end{array}\right](v \mid \tau)=-e^{i \pi \alpha} d\left[\begin{array}{l}
\alpha \\
\beta
\end{array}\right](v \mid \tau) .
\end{aligned}
$$

Consistency of the index formula requires that $I\left(g_{1}, g_{2} ; \tau\right)$ is invariant under the subgroup of the modular group which preserves the twists. Only then is it possible to obtain a modular invariant index by summing contributions from different twisted sectors. Under a modular transformation $\tau \rightarrow \Lambda \cdot \tau=(a \tau+b / c \tau+d)$, we have [31]

$$
d\left[\Lambda\left(\begin{array}{c}
\alpha \\
\beta
\end{array}\right)\right](0 \mid \Lambda \cdot \tau)=\varepsilon(\Lambda) d\left[\begin{array}{l}
\alpha \\
\beta
\end{array}\right](0 \mid \tau)
$$

where $\Lambda\left(\begin{array}{l}\alpha \\ \beta\end{array}\right)$ denotes the matrix $\Lambda$ acting on the vector $\left(\begin{array}{c}\alpha \\ \beta\end{array}\right)$ and $\varepsilon(\Lambda)$ is a twelfth root of unity which depends only upon $\Lambda$. If $\Lambda$ preserves the twists then $\Delta_{1}$ and $\Delta_{2}$ defined by

$$
\Delta_{1}=(a-1) \alpha+b \beta, \quad \Delta_{2}=c \alpha+(d-1) \beta
$$

are integers. From (4.9)-(4.12) we find that

$$
d\left[\begin{array}{l}
\alpha \\
\beta
\end{array}\right](\Lambda \cdot v \mid \Lambda \cdot \tau)=\varepsilon(\Lambda) e^{l \pi\left[\phi(\alpha, \beta ; \Lambda)+c v^{2} /(c \tau+d)\right]} d\left[\begin{array}{l}
\alpha \\
\beta
\end{array}\right](v \mid \tau),
$$

where $\Lambda \cdot v=v /(c \tau+d)$ and

$$
\phi(\alpha, \beta ; \Lambda)=\Delta_{1}+\Delta_{2}+\Delta_{1} \Delta_{2}+\alpha \Delta_{2}-\beta \Delta_{1}
$$

is a phase which depends on the twists, $\alpha$ and $\beta$, and on $\Lambda$, but does not depend on $v$. We also note that only a term quadratic in $v$ appears in the phase.

Arguments similar to those used in Sect. 2 show that $I\left(g_{1}, g_{2} ; \Lambda \cdot \tau\right)=I\left(g_{1}, g_{2} ; \tau\right)$ provided the sum of phases from all the terms in (4.5) vanishes after we integrate over $M_{g_{1} g_{2}}$. First consider the $v$-independent terms. The sum of the phases (4.15) represents the obstruction that comes only from the twists and is independent from the background. In other words it is the same obstruction that is encountered on orbifolds without background fields. Thus we can use the results of the detailed analysis of [27] and [28], to conclude that the level matching condition (3.44) is 
the necessary and sufficient condition for this part of the phase to vanish ${ }^{11}$. The $v$-dependent part of the phase yields

$$
\exp \left[\frac{i \pi c}{c \tau+d}\left(\operatorname{Tr} F^{2}-\operatorname{Tr} R^{2}\right)\right]
$$

where $R$ and $F$ are the full curvatures of $T M$ and $E$ restricted to the fixed point surface $M_{g_{1} g_{2}}$. Once more, if $\operatorname{Tr} F^{2}-\operatorname{Tr} R^{2}=d H$, for some 3-form $H$ on $M_{g_{1} g_{2}}$, then the contribution of this phase to the modular transformation of (4.5) vanishes.

We have thus established that the consistency of the index formula (4.5) on an abelian orbifold requires that the level matching condition is satisfied and the free parts of the Pontryagin classes of $T M$ and $E$, restricted to the fixed point surface, $M_{g_{1} g_{2}}$, satisfy $p_{1}\left(\left.T M\right|_{M_{g_{1} g_{2}}}\right)=p_{1}\left(\left.E\right|_{M_{g_{1} g_{2}}}\right)$.

We can compare this result with the full $\lambda$-class on the orbifold which was given in (3.47). As previously the index formula detects only a part of this class: the free part of the first term, which is quadratic in curvatures, and the last term which is quadratic in twists. Neither the torsion part of $\frac{1}{2}\left[p_{1}\left(\left.T M\right|_{M_{g_{1} g_{2}}}\right)-\right.$ $\left.p_{1}\left(\left.E\right|_{M_{g_{1} g_{2}}}\right)\right]$ nor the cross-term between the curvatures and twists, which may also be present in $\lambda$, cause any inconsistencies in the index formula. However, they must vanish for the Dirac-Ramond operator to exist.

\section{Conclusions}

We have shown that the existence of string structure on an abelian orbifold is equivalent to the absence of local and global anomalies in the corresponding $\sigma$-model, only after one requires that the string structure admits an action of a circle or more precisely of $\operatorname{Diff}^{+}\left(S^{1}\right)$. This gives a geometric interpretation of the level matching condition. Some part of the obstruction to the existence of a string structure can be detected by the index formula for the Dirac-Ramond operator. This formula can be derived by formal application of the fixed point theorem or equivalently by a high temperature expansion of a suitable path integral. Validity of both methods requires that not only a string structure exists, but also that it has a well-defined $S^{1}$ symmetry. Therefore one expects that the $\lambda$-class should be detectable by the index formula. We saw that it is indeed the case. However, it is only the localizable part of $\lambda$ that can be probed in this way. On a smooth manifold this restricts us to the free part of the cohomology class of $\lambda$, but if the manifold has singularities, as in the case of orbifolds, then even an infinitesimally small torus localized at the singular point can detect the torsion part of $\lambda$, that is the level matching condition.

It is known that on nonabelian orbifolds the level matching condition gives only a part of the $\lambda$-class and thus does not guarantee the absence of global anomalies $[27,28]$. It would be interesting to understand in this case what is the interpretation of $\lambda$ in terms of the geometry of loop bundles.

${ }^{11}$ To prove the necessity one must use that $\Gamma$ is abelian 
Acknowledgements. We would like to thank D. Freed, C. Vafa and E. Witten for discussions.

\section{References}

1. Witten, E.: Global anomalies in string theory. In: Anomalies, geometry and topology. Bardeen, W., White, A. (eds.). pp. 61-99 Singapore: World Scientific 1985

2. Schellekens, A. N., Warner, N. P.: Anomalies and modular invariance in string theory. Phys. Lett. 177B, 317-323 (1986); Anomaly cancellation and self-dual lattices. Phys. Lett. 181B, 339-343 (1986)

3. Schellekens, A. N., Warner, N. P.: Anomalies, characters and strings. Nucl. Phys. B287, 317-361 (1987)

4. Pilch, K., Schellekens, A. N., Warner, N. P.: Path integral calculation of string anomalies. Nucl. Phys. B287, 362-380 (1987)

5. Witten, E.: Elliptic genera and quantum field theory. Commun. Math. Phys. 109, 525-536 (1987)

6. Landweber, P., Stong, R. E.: Circle actions on spin manifold and characteristic numbers. Rutgers preprint (1985)

7. Ochanine, S.: Sur les genre multiplicatifs définis par des intégrals elliptique. Topology 26, 143-151 (1987)

8. Chudnovsky, D. V., Chudnovsky, G. V.: Elliptic modular forms and elliptic genera. Columbia University preprint (1985)

9. Zagier, D.: Note on the Landweber-Stong elliptic genus, preprint

10. Witten, E.: Fermion quantum numbers and Kaluza-Klein theory. In the Proceedings of the 1983 Shelter Island Conference on Quantum Field Theory and the Foundations of Physics. Khuri N. et al. (eds.). Cambridge, MA; MIT Press 1985

11. Witten, E.: The index of the Dirac operator in loop space. Princeton preprint PUPT-1050 (1987)

12. Taubes, C.: $S^{1}$ actions and elliptic genera. Harvard preprint (1987)

13. Atiyah, M. F., Singer, I. M.: The index of elliptic operators IV, V. Ann. Math. 93, 119-149 (1971)

14. Killingback, T.: World-sheet anomalies and loop geometry. Nucl. Phys. B288, 578-588 (1987)

15. Pressley, A., Segal, G. B.: Loop Groups. Oxford: Oxford University Press 1986

16. Moore, G., Nelson, P.: Anomalies in nonlinear sigma models. Phys. Rev. Lett. 53, 1519-1522 (1984)

17. Witten, E.: Global gravitational anomalies. Commun. Math. Phys. 100, 197-229 (1985)

18. Freed, D. S.: Determinants, torsion, and strings. Commun. Math. Phys. 107, 483-513 (1986)

19. Bismut, J.-M., Freed, D. S.: The analysis of elliptic families: Metrics and connections on determinant bundles. Commun. Math. Phys. 106, 159-176 (1986); The analysis of elliptic families: Dirac operators, eta invariants, and holonomy theorem of Witten. Commun. Math. Phys. 107, 103-116 (1986)

20. Green, M. B., Schwarz, J. H.: Anomaly cancellations in supersymmetric $d=10$ gauge theory and superstring theory. Phys. Lett. 149B, 117 (1984)

21. See, e.g., Alvarez-Gaumé, L., Ginsparg, P.: The structure of gauge and gravitational anomalies. Ann. Phys. 161, 423-490 (1985)

22. Hull, C., Witten, E.: Supersymmetric sigma models and the heterotic string. Phys. Lett. 160B, 398-402 (1985)

23. Atiyah, M. F., Bott, R.: A Lefschets fixed point formula for elliptic complexes: II. Applications. Ann. Math. 88, 451-491 (1968); (-), Hirzebruch, F.: Spin manifolds and group actions. In: Essays in topology and related subjects. Berlin, Heidelberg, New York: Springer 1970, pp. 18-28

24. Alvarez, O., Killingback, T., Mangano, M., Windey, P.: String theory and loop space index theorems. Commun. Math. Phys. 111, 1-10 (1987)

25. Wen, X. G., Witten, E.: Electric and magnetic charges in superstring models. Nucl. Phys. 261, 651-677 (1985)

26. Dixon, L., Harvey, J., Vafa, C., Witten, E.: Strings on orbifolds. Nucl. Phys. B261, 678-686 (1985); Strings on orbifolds (II). Nucl. Phys. B274, 285-314 (1986)

27. Vafa, C.: Modular invariance and discrete torsion on orbifolds. Nucl. Phys. B273, 592-606 (1986)

28. Freed, D. S. Vafa, C.: Global anomalies on orbifolds. Commun. Math. Phys. 110, 349-390 (1987) 
29. Atiyah, M. F., Bott, R.: The moment map and equivariant cohomology. Topology 23, 1-28 (1984)

30. $\mathrm{Li}, \mathrm{K} .:$ Character-valued index theorems in supersymmetric string theories. MIT preprint CTP-1460 (1987)

31. Alvarez-Gaumé, L., Moore, G., Vafa, C.: Theta functions, modular invariance, and strings. Commun. Math. Phys. 106, 1-40 (1986)

Communicated by A. Jaffe

Received August 5, 1987 